\title{
CASPAM: a triple modality biosensor for multiplexed imaging of caspase network activity
}

Martín Habif ${ }^{1,2, \dagger}$, Agustín A. Corbat $^{1, \dagger}{ }^{\dagger}$ Mauro Silberberg ${ }^{1}$, and Hernán E. Grecco ${ }^{1,3, *}$

${ }^{\dagger}$ Authors contributed equally to this work

${ }^{1}$ Department of Physics, FCEN, University of Buenos Aires and IFIBA, CONICET, Buenos Aires, Argentina

${ }^{2}$ Current Address: Instituto de Biología Celular y Neurociencia (IBCN) "Prof. EDUARDO DE ROBERTIS", Facultad de Medicina, Universidad de Buenos Aires and CONICET, Ciudad de Buenos Aires, Argentina

${ }^{3}$ Department of Systemic Cell Biology, Max Planck Institute of Molecular Physiology, Dortmund, Germany.

*Corresponding author: hgrecco@df.uba.ar 


\section{Supplementary Information}

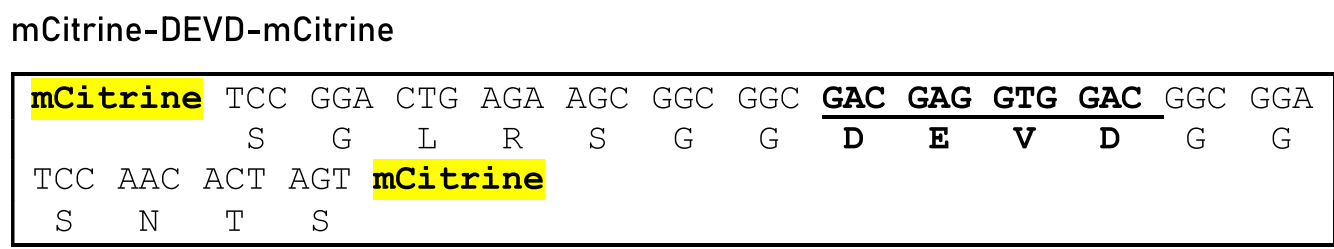

mCitrine-GGGSGGG-P2A-tagBFP

\begin{tabular}{|ccccccccccccccc|}
\hline mCitrine & GGA & GGA & GGA & AGC & GGA & GGA & GGA & GCT & ACT & AAC & TTC & AGC & CTG \\
CTG & AAG & CAG & GCT & GGA & GAC & GTG & GAG & GAG & AAC & CCT & GGA & CCT & tagBFP \\
\hline L & K & Q & A & G & D & V & E & E & N & P & G & P & \\
\hline
\end{tabular}

tagBFP-2xIETD-Cerulean

\begin{tabular}{|ccccccccccccccccc}
\hline tagBFP & TCC & GGA & CTG & AGA & AGC & GGC & GGC & ATC & GAG & ACC & GAC & GGC & GGC \\
& S & G & L & R & S & G & G & I & E & T & D & G & G \\
ATC & GAG & ACC & GAC & GGC & GGA & TCC & AAC & ACT & AGT & Cerulean & & \\
\hline I & E & T & D & D & G & G & S & N & T & S & & & & \\
\hline
\end{tabular}

Cerulean-GGGGSGGGG-T2A-mCherry

\begin{tabular}{|ccccccccccccccc|}
\hline Cerulean & GGA & GGA & GGA & AGC & GGA & GGA & GGA & GAG & GGC & AGA & GGA & AGT & CTG \\
CTA & ACA & TGC & GGT & GAC & GTC & GAG & GAG & AAT & CCT & GGA & CCT & mCherry & \\
\hline I & T & C & G & D & V & E & E & N & P & G & P & & \\
\hline
\end{tabular}

mCherry-LEHD-mKate2

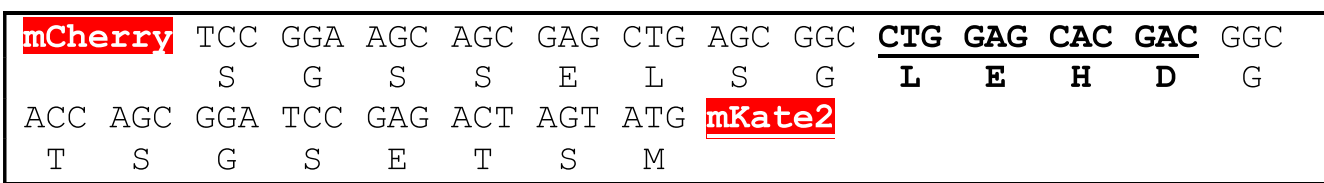

Supplementary Figure 1: DNA and corresponding amino acid sequences of caspase cleavage sites and $2 \mathrm{~A}$ peptides used in this study for mCitrine-DEVD-mCitrine, P2A, tagBFP-2xIETD-Cerulean, T2A and mCherry-LEHD-mKate2 respectively. Underlined sequences encode functional amino acids. For clarifying purposes, the four Nor C-terminal aminoacids of each fluorescent protein were included in each sequence. 
b
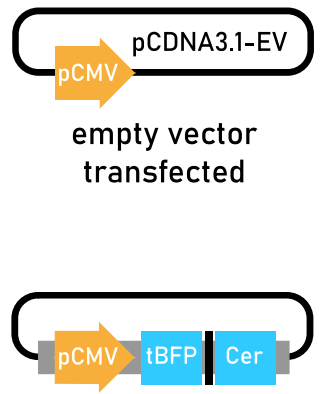

sCas9-BP

C

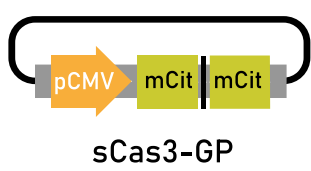

d

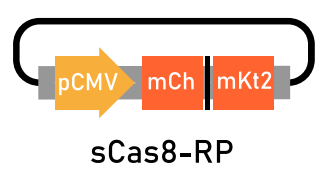

$X:$ BP $530 / 30$

Y: BP 450/50

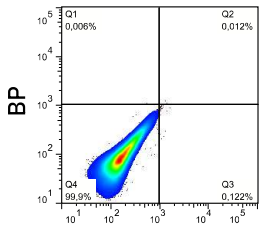

GP

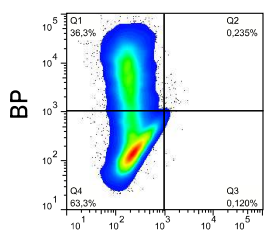

GP
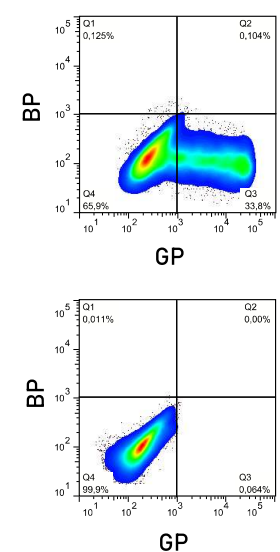

$X: B P 610 / 20$

Y: BP 450/50

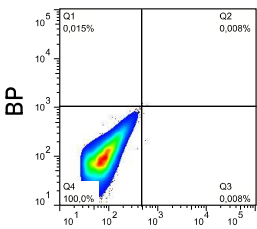

RP
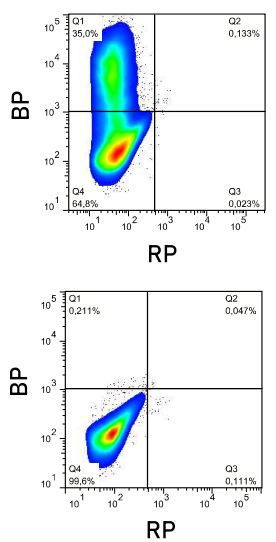

RP

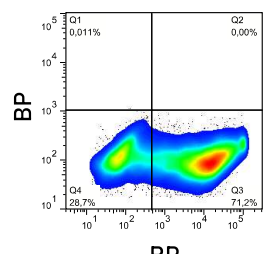

$X: B P 610 / 20$

Y: BP $530 / 30$

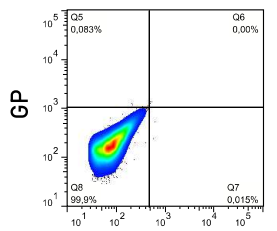

$\mathrm{RP}$
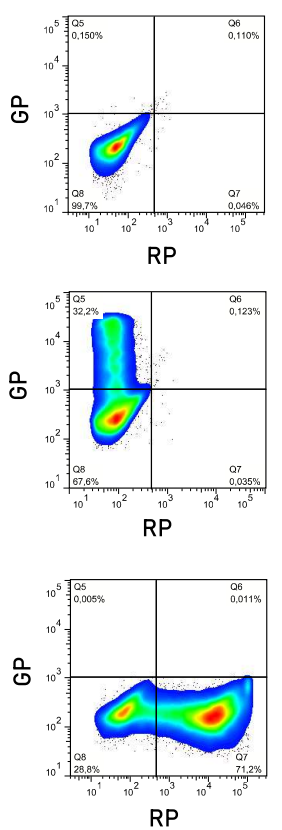

Supplementary Figure 2: Flow cytometric detection of single biosensor expression. Hela cells were transfected with plasmids encoding a single FPA caspase activity biosensor, as detailed in Materials \& Methods. Briefly, transfected cells were grown for $24 \mathrm{~h}$ prior to harvest and analysis by flow cytometry. Samples were acquired using FACS-Aria Fusion cell cytometer, and a minimum of 20,000 events were collected for each sample. As seen from the representative histograms only two cell populations were detected: cells expressing one biosensor and untransfected/non biosensor-expressing cells (percentages are indicated on the plots). (a) pcDNA3.1 empty vector, (b) sCas9-BP, (c) sCas3-GP, (d) sCas8-RP. Single biosensor expression assessments served as single-color compensation controls to calculate the amount of compensation required for the co-expression determinations. 
a

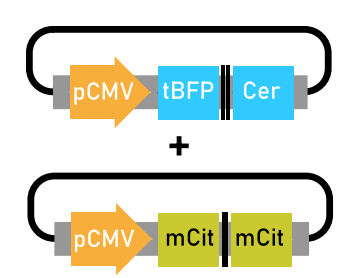

b

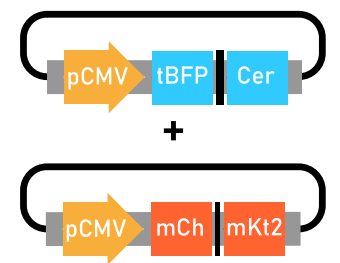

C

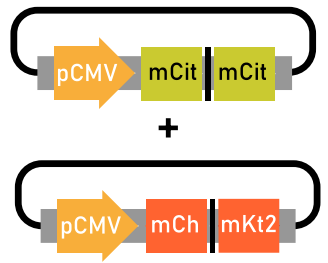

X: BP $530 / 30$

Y: BP 450/50
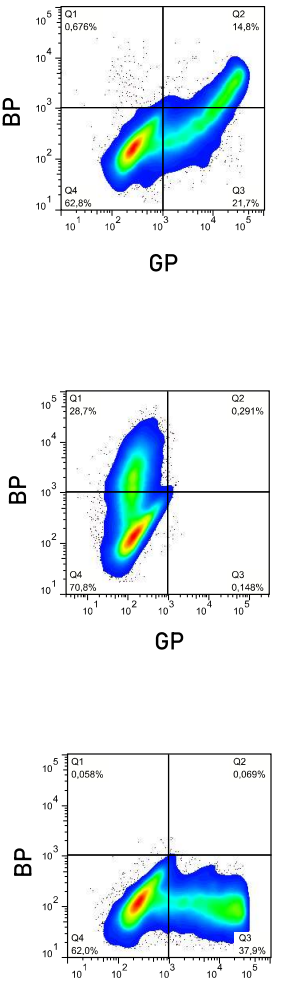

GP
$X$ : BP $610 / 20$

Y: BP 450/50

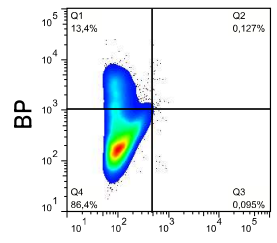

RP
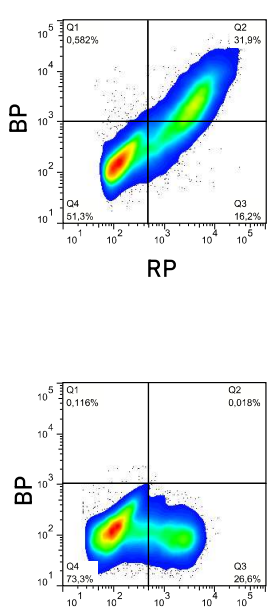

RP
$X$ : BP $610 / 20$

Y: BP 530/30
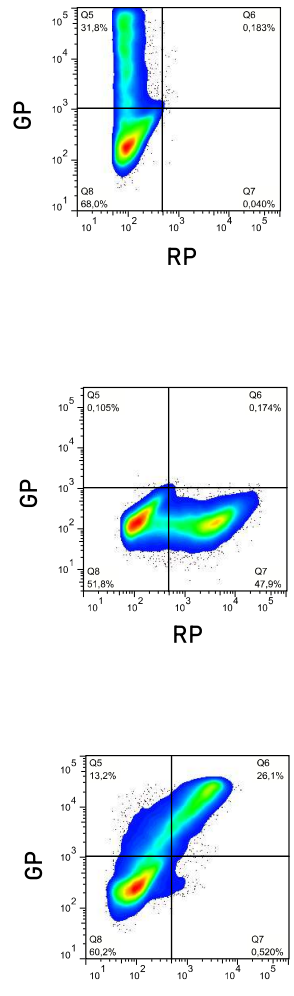

RP

Supplementary Figure 3: Flow cytometric detection of single cell dual biosensor co-expression. Hela cells were transfected with different combinations of two plasmids, each one encoding a single FPA caspase activity biosensor in 1:1 $(\mathrm{w} / \mathrm{w})$ mixes, as detailed in Materials \& Methods. As seen from the representative histograms, all major cell populations were detected: cells expressing one of the biosensors, both biosensors, and neither biosensor (percentages are indicated on the plots). Cells expressing the biosensors are easily distinguished from those untransfected/non-biosensor-expressing cells. (a) sCas9-BP+sCas3-GP, (b) sCas9$\mathrm{BP}+\mathrm{sCas} 8-\mathrm{RP}$, (c) sCas3-GP+sCas8-RP. 

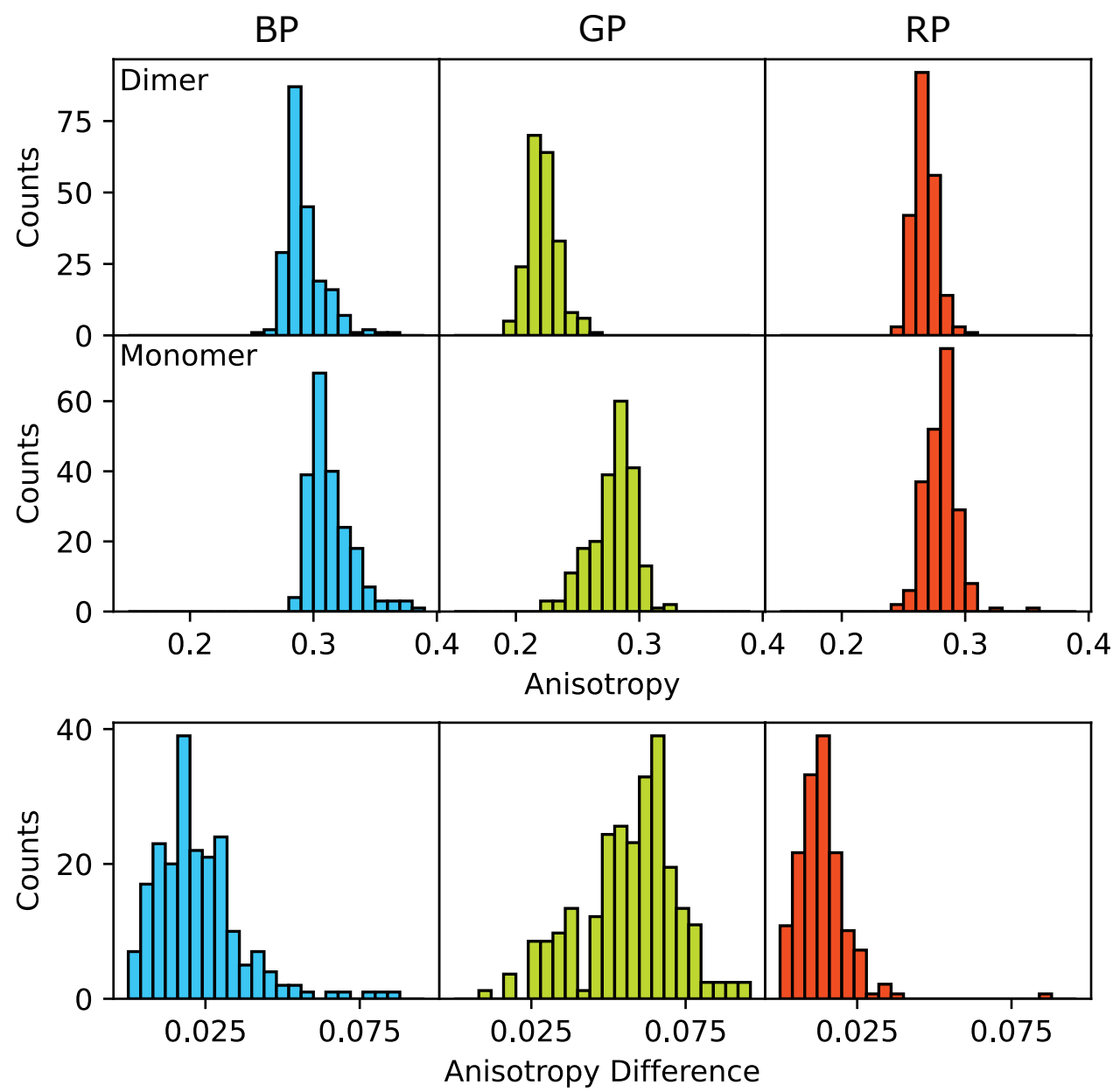

Supplementary Figure 4: Histograms for dimer, monomer anisotropies and their difference for each biosensor in CASPAM. On the top rows we can appreciate Dimer and Monomer Anisotropy histograms of all cells transfected with CASPAM. Although dimer and monomer anisotropy look close to one another, there is always a positive and appreciable difference between them, as shown in the bottom row histogram. Variability in anisotropies can be generated by lack of complete cleavage or errors in background subtraction among other sources of noise.

Supplementary video V1. Fluorescence intensity and anisotropy video corresponding to each channel of the cell presented in Figure 5. Anisotropy curve is also plotted below with a dashed line showing the actual frame. 